\title{
Web based Weather Monitoring System using Wireless Sensor Node
}

\author{
Immanuel J. \\ Department of Instrumentation \\ Technology \\ Gulbarga University \\ P.G. Centre, Yeragera \\ Raichur, KARNATAKA, INDIA
}

\author{
Rekha Patil \\ Department of Computer \\ Science \\ Gulbarga University \\ P.G. Centre, Yeragera \\ Raichur, KARNATAKA, INDIA
}

\author{
Sudheer L. S. \\ Department of Instrumentation \\ Technology \\ Gulbarga University \\ P.G. Centre, Yeragera \\ Raichur, KARNATAKA, INDIA
}

\author{
Parvathi C. S. \\ Department of Instrumentation Technology \\ Gulbarga University P.G. Centre, Yeragera \\ Raichur, KARNATAKA, INDIA
}

\author{
Bhaskar P. \\ Department of Instrumentation Technology \\ Gulbarga University P.G. Centre, Yeragera \\ Raichur, KARNATAKA, INDIA
}

\begin{abstract}
Wireless sensor node refers to a group of specifically designed, fabricated, dispersed and dedicated sensors for monitoring and recording the physical conditions of the environment and organizing the collected data at a location. Internet of things (IoT) is a network of physical devices and it is tagging our day-to-day objects with our lives. To access data from the sensor node through web page is much easier way. In this paper an attempt is made to design web based approach to monitor weather parameters and their conditions. The webpage is an interface between user and the weather monitoring system. To acquire and transmit weather parameters, wireless sensor node is designed and fabricated using Arduino UNO board. Also, to connect the sensor node to the internet to send the data to the cloud an ESP8266 Wi-Fi device is interfaced with Arduino. The weather parameters such as atmospheric temperature, humidity, sunlight (intensity), and rainfall are measured and transmitted over the internet. A webpage is created to display the present and past values of the measured parameters. The page also displays the current status of the parameters being measured. This page is linked with the website so that the parameters can be accessed over the internet.
\end{abstract}

\section{Keywords}

Arduino, IoT, Monitoring, Sensor node, Weather, Webpage, Wireless.

\section{INTRODUCTION}

The past three decades have seen the introduction of a technology that has radically changed the way in which the things are analyzed and controlled. There is no doubt that our daily lives are significantly affected by electronic engineering technology. In each and every field of science and technology the people are exposed with sophisticated electronic devices and systems. Electronics is characterized by reliability, low power dissipation, low weight and volume, and low cost. To achieve such characteristics, embedded systems were developed. Also with embedded system sensors were built to make reliable and efficient applications in the field of measurement and control. Some of the researchers reported the use of wireless sensor nodes.

Mohamed Amine Kafi et al., reported the wireless sensor network (WSN) based intelligent transport system. They presented a comprehensive review on WSN based ITS solutions and they classified from application prospective i.e., vehicle traffic in urban areas[1].

With advancement of automation technology, life has become simpler and easier in almost all aspects. Nowadays, automatic systems are being preferred over manual operating system worldwide. The rapid increase in the number of internet users over the past decade has made internet a part of life, and IoT is the latest internet technology emerging in today's world. Internet of things is a developing network of everyday where all the objects from industrial machine to consumer goods can share data and complete tasks while users are occupied with different activities. IoT is a system that uses computers or mobile devices to control basic functions of home and its features automatically through internet from anywhere around the world.

M.Lavanya P. et al reported Raspberry Pi based system running with Linux OS coded in $\mathrm{C}++$ retrieves the temperature as well as humidity readings and sends to the internet[2]. Andrea Zanella et al presented literature on IoT technologies and analyzed the solutions currently available for implementation of urban IoTs. They discussed technologies are close to being standardized, and industry players are already active in the production of devices that take advantage of these technologies to enable the applications of interest. They concluded that the technologies have reached a level of maturity that allows for the practical realization of IoT solutions and services, starting from field trials that will hopefully help clear the uncertainty that still prevents a massive adoption of the IoT paradigm[3].

Mohd Ezwan Jalil, reported that the recent technologies in wireless communications and electronics have brought the vision of WSN which have increased the growth of low cost, low power and multi-functional sensors, which are small in size and can communicate in short range. The author described that each node consists of microcontrollers, memory and transceiver. Microcontrollers are used to execute task, data processing and assist the functionality of other components in the sensor node[4]. Ding Libo and Wang Xuehmi reported the temperature and humidity monitoring system in scientific experiment and quality testing laboratories. In order to supervise and record the temperature and humidity of geographical distributed constant temperature and humidity testing laboratories, a web-based monitoring 


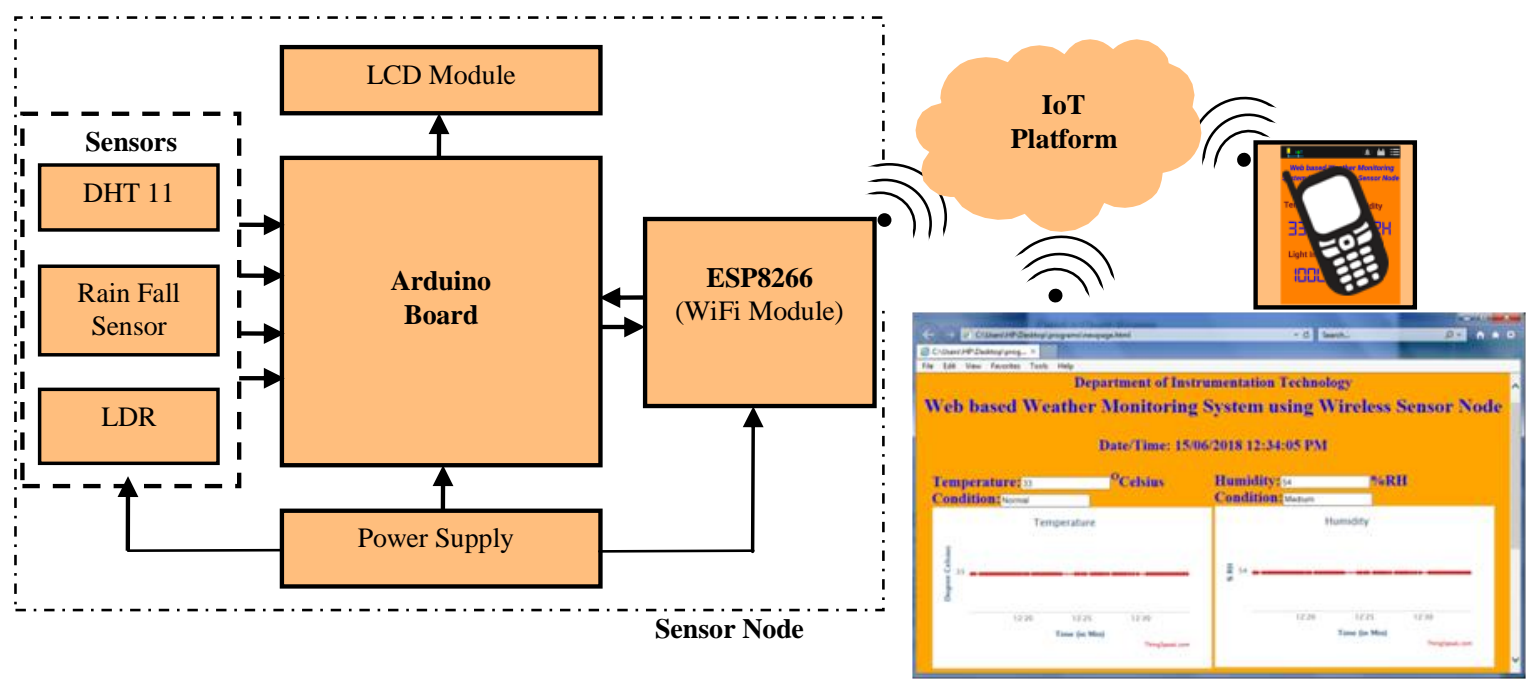

Fig 1. Block diagram of web based weather monitoring system using wireless sensor node

system was developed. The MCU collects the data from the sensors and transmitting them to a remote data server[5]

Ali Akbar Siddiqui et al reported the temperature monitoring system using Raspberry Pi and interfaced with four different sensor nodes, ZigBee module is used for wireless data transmission from sensor node to Raspberry Pi. Implementation of such system on Raspberry $\mathrm{Pi}$ is cost effective and they simulated whole system on porteus based environment and results were comprehensively accurate and resembled to that of the survey of boilers[6].

Akash, and Amit Birwal reported the IoT based temperature and humidity monitoring system for agriculture application. They employed Raspberry Pi and DHT11 sensor for temperature and humidity measurement, which reduces the human interference in taking care of these parameters manually. The key features of their project are to measure and save the data to the internet web for later use of the same[7]. Bulipe Srinivas Rao, et al proposed in their paper the advanced solution for monitoring the weather conditions at a particular place and makes the information visible anywhere in the world based on IoT Technology. The system deals with monitoring and controlling the environmental conditions like temperature, relative humidity, light intensity and carbon monoxide level with sensors and send the information to the web page and then plot the sensor data as graphical statistics[8]. From the literature it is observed that the IoT is now the basic platform for the measurement and control applications and very less work has been reported in the implementation of wireless sensor node with web page to send the data over the internet to make it available worldwide.

\section{INSTRUMENTATION}

The Figure 1 shows the block diagram of proposed web based weather-monitoring system. It consists of wireless sensor node, web server/IoT platform and the webpage.

\subsection{Wireless sensor node}

A wireless sensor node is an intelligent device which senses the one or more physical parameters of interest and transmits the sensor data to the web server. It consists of the DHT11, rain sensor, LDR, Arduino UNO board, and ESP8266 module. Figure 2 shows the DHT11 which is a composite sensor containing a calibrated digital signal output of the temperature and humidity. Low cost, long-term stability, relative humidity and temperature measurement, excellent quality, fast response, strong anti-interference ability, long distance signal transmission, digital signal output, and precise calibration are the salient features of this sensor. The sensor includes a resistive sense of wet components and NTC temperature measurement devices, and connected with a high-performance 8-bit microcontroller.

The applications of DHT11 are HVAC, dehumidifier, testing and inspection equipment, consumer goods, automotive, automatic control, data loggers, weather stations, home appliances, humidity regulator, medical and other humidity measurement and control. It measures relative humidity with 16-bit resolution and accuracy of $\pm 5 \mathrm{RH}$. Also, it measures temperature with 16-bit resolution [9].

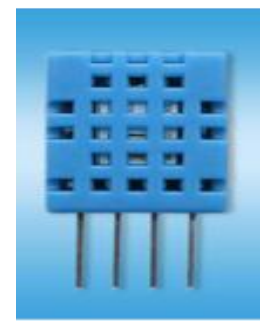

\section{Fig 2. DHT11 Sensor}

Figure 3 shows the rain sensor. The rain sensor detects water that completes the circuits on its sensor boards printed leads when it falls on it. The sensor board acts as a variable resistor that will change from $100 \mathrm{k}$ ohms when wet to $2 \mathrm{M}$ ohms when dry. In short wetter the board more the current that will be conducted [10]. 


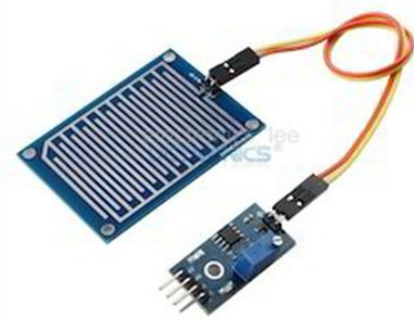

Fig.3. Rain Sensor

Figure 4 shows light dependent resistor (LDR) sensor. This resistor works on the principle of photo conductivity. It is nothing but, when the light falls on its surface, then the material conductivity reduces. These devices depend on the light, when light falls on the LDR then the resistance decreases, and increases in the dark. When a LDR is kept in the dark place, its resistance is high and, when the LDR is kept in the light its resistance will decrease [11].

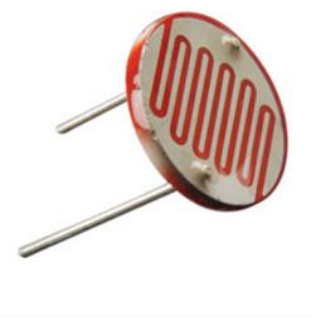

Fig 4. LDR Sensor

The Arduino UNO board is used to design wireless sensor node. The sensors and WiFi module are interfaced with the board. The board acquires the sensor data and sends the data to the Web Server (IoT platform). The ESP8266 module is a low cost WiFi module which is used to connect the sensor node to the internet. The LCD module is also interfaced with the node and parameters are displayed on the LCD locally. With known SSID and password the node logs on to the internet.

\subsection{Web server (IoT platform)}

The thingspeak server from Math works Inc. is created for the present work. This server has one channel and four fields for four parameters. This channel offers for every $15 \mathrm{sec}$ data is stored on to the cloud. The server has an option to recover the previously stored data.

\subsection{Webpage}

The webpage created for the present application is used to access the sensor data sent by the sensor node over the internet. This html document file is linked with the website so that the instrument data can be accessed anywhere from the world. This page contain various controls such as text boxes to display parameters such as Temperature, Humidity, rain fall and light intensity.

The parameter variations are also visualized from the graphical representation of the said parameters. This helps in data analysis and predicting the weather conditions. The weather conditions are displayed on the webpage by analyzing the current incoming data from the sensor node. This web page is linked with our department website http://instrumentation.my-free.website, which is created to demonstrate the present application. By clicking on the link, the webpage opens up and displays weather parameters with their current conditions. Once the sensor node starts acquiring the, data is stored in the web server.

This web server (cloud) is created in the Mathworks Inc, Thingspeak IoT platform allows huge data storage since instruments started to acquiring the parameters. From each channel on the web server the data can be accessed in the web page and displayed on it. Each data is displayed on the web page with current date and time information.

Hence this system can be used as data logger system. Apart from normal tags to refresh page for every $15 \mathrm{sec}<$ meta $>$ tag is used. And to display four parameters <iframe $>$ tag is used to create frames. By using <table> tag two rows and two columns table is created and each frame is made to fit in one cell along with corresponding values. In the $\langle$ script $\rangle$ tag the feed is accessed by creating function called myfunction and this function is continuously refreshes the page and after refresh the data is displayed. This function contains http requests and by GET method the URL is accessed from Thingspeak. After getting response from URL it will access the feed from the Thingspeak. This feed contains fields, charts, and date and time information.

\section{METHODOLOGY}

The wireless sensor node designed for the present application acquires the environmental parameters such as temperature, humidity, sun light, and rainfall. The sensor node contains Arduino UNO board which has Atmel microcontroller on it. The on-chip ADC of microcontroller acquires the voltages corresponding to the above said parameters from the respective sensors through analog input channels. A WiFi module (ESP8266) is interfaced to sensor node connects to internet by the firmware programmed through microcontroller (with known SSID \& password).

Once the sensor node is connected to internet (wireless network), the measured sensor data are sent to the web server, where the data is stored or recorded continuously in the cloud. The stored data is accessed by webpage designed for the present application. All the measured parameters are displayed on the web page with their current status as indicators. The webpage can be linked with the website so that the data can be accessed anywhere from the world. The webpage can be best viewed on internet explorer version 8 or higher.

The flow chart of the program for the present application is shown in Figure 5. The software is developed in the Arduino IDE. After initialization of constants, variables the arduino board and wifi module logs on to the internet by known SSID and password which are pre-programmed in the board. The software for acquiring and sending data to the IoT platform is written and loaded into the arduino board.

\section{EXPERIMENTAL RESULTS}

The figure 6(a) \& (b) show the screen shots of webpage created for the present application. The data from the sensor node are stored on to the web server. The webpage accesses the data from the web server and displays in the form of numerical value as well as graphical visualization. The Graph is displayed for each parameter.

Also, the current status of temperature, humidity, rain \& light intensity are displayed by checking the current value with the pre-set value. If temperature is between $0^{\circ} \mathrm{C}$ to $20^{\circ} \mathrm{C}$ the webpage displays as "Low", for $20^{\circ} \mathrm{C}$ to $34^{\circ} \mathrm{C}$ the webpage 


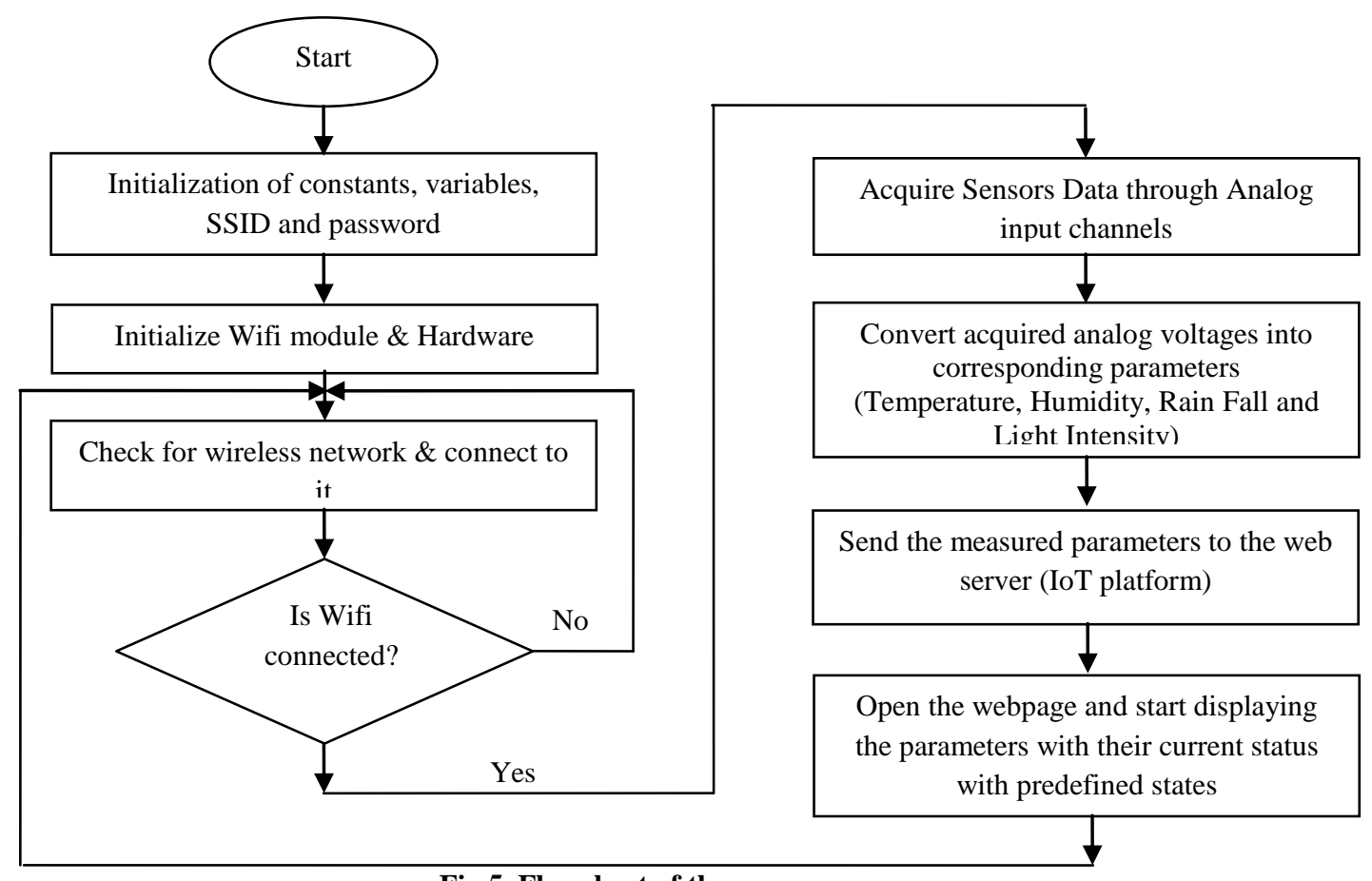

Fig 5. Flowchart of the program

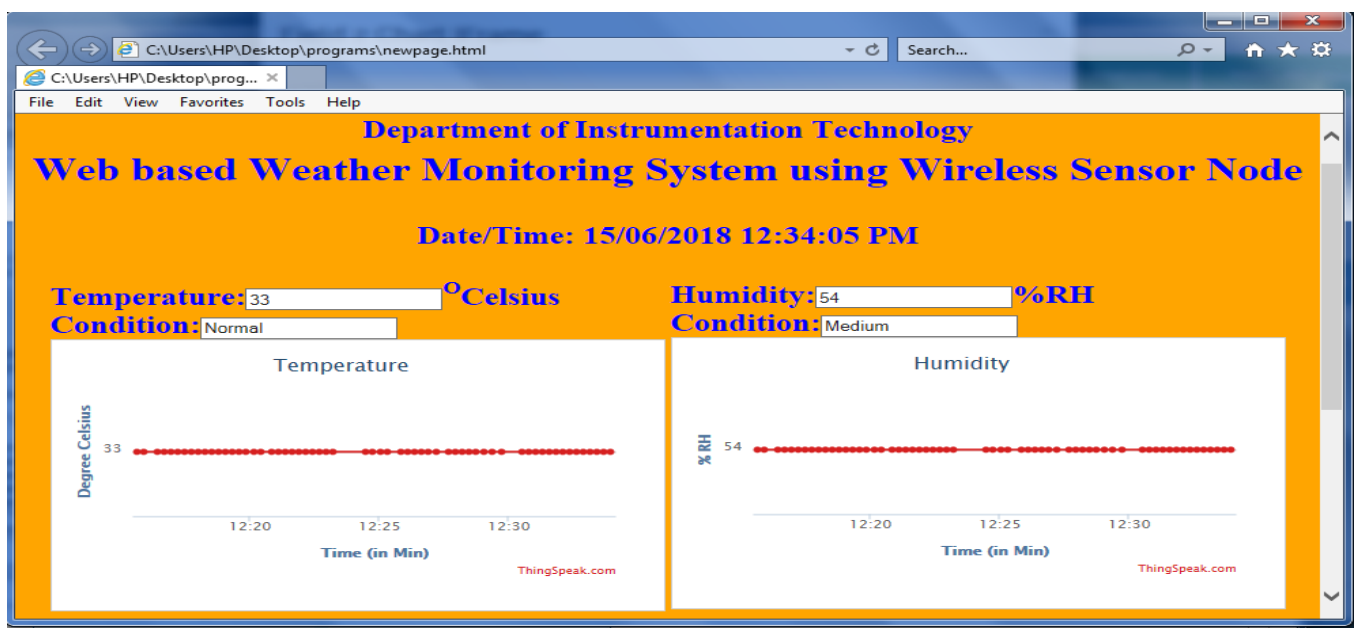

Fig 6(a). Screen-shot of webpage

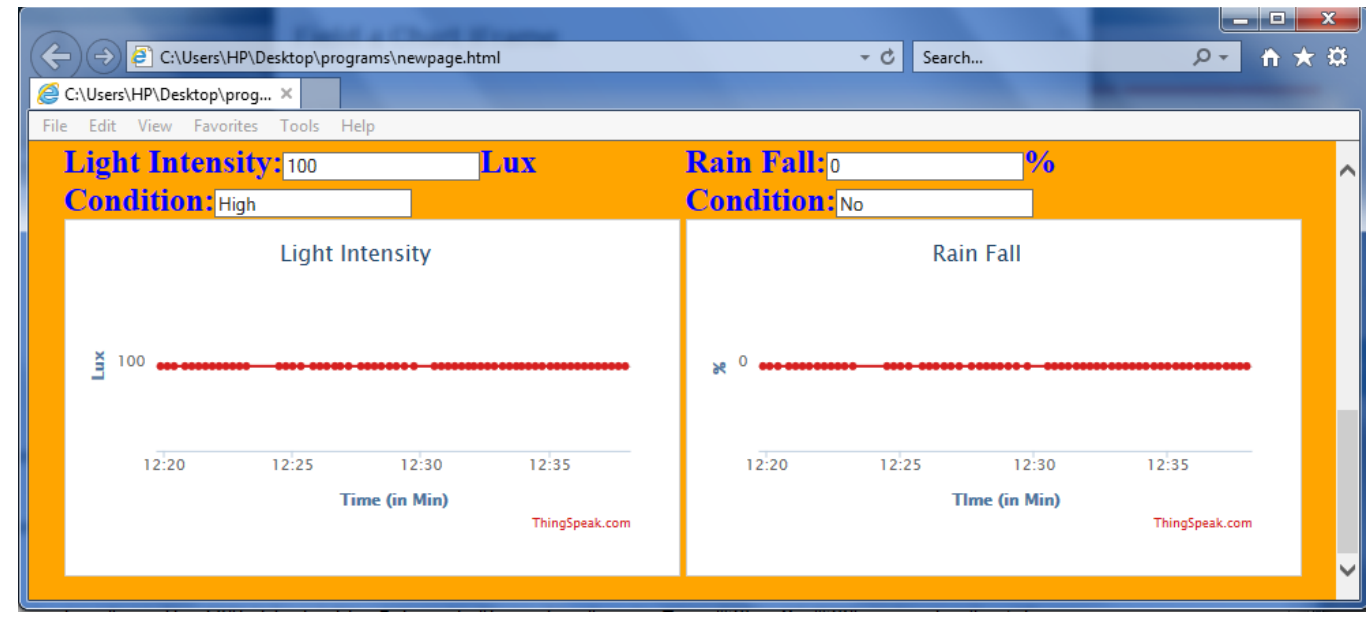

Fig 6 (b). Screen-shot of graphs being displayed 
displays as "Medium" and for above $34^{\circ} \mathrm{C}$ it displays as "High". Similarly, for humidity $0-30 \% \mathrm{RH}$ as "Low", for $30 \% \mathrm{RH}$ to $60 \% \mathrm{RH}$ as "Medium" and above $60 \% \mathrm{RH}$ as "High". The light intensity from 0-50Lux is displayed as "Low", 50-100Lux as "Medium" and above 100Lux as "High". The rainfall is also indicated by three conditions such as "No", "Medium" and "High".

Figure 7 shows the mobile screen shot of the same. The android application is developed by using Virtuino application. This provides all the controls to display Temperature, Humidity, Light Intensity and rainfall. It connects to the thingspeak server and gets the data and displays it on the layout.

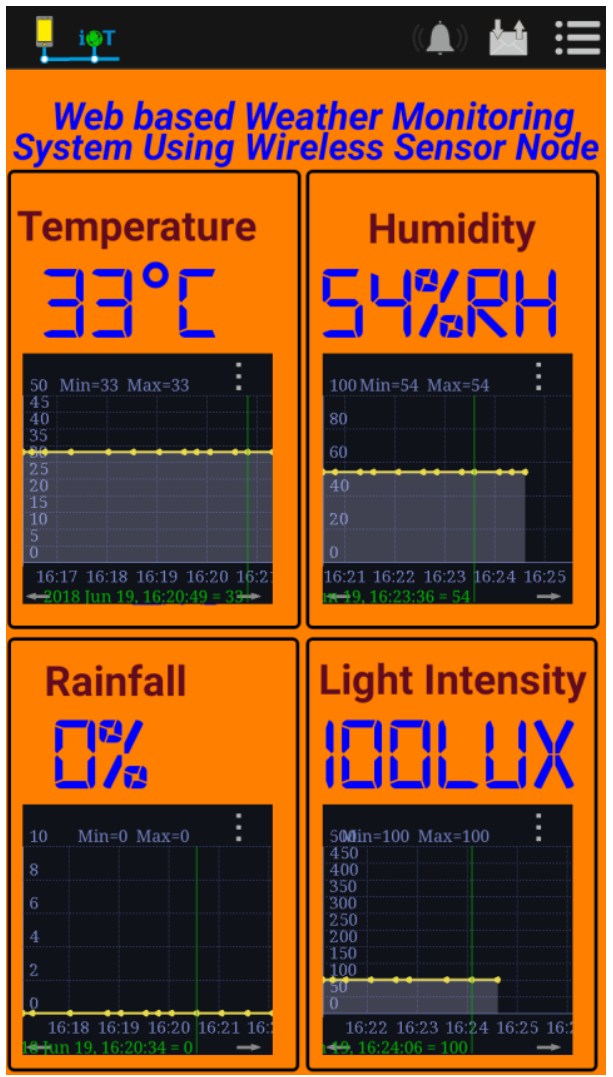

Fig 7. Virtuino app mobile Screen-shot

\section{CONCLUSIONS}

From the results, it is observed that, the system effectively measures and displays the parameters such as ambient/atmospheric temperature, humidity, rainfall and light intensity. The hardware is connected to the internet and through webpage the data can be analyzed and linked with website. Through website the system data can be accessed from anywhere in the world. Further this IoT system can also be used for Agriculture applications such as green house monitoring. With few modifications in the hardware and software, the said parameters can also be controlled in the predefined area and applied to in climate control. In this system the data available in the cloud can be accessed by the mobile application which stores the data and be analysed.

\section{ACKNOWLEDGEMENTS}

The Authors are very grateful to Mathworks Inc. USA for providing free IoT platform, and Websitebuilder.com for their support to build our own website. Also, to Mr. Laxmikant
Dixit, Lecturer, Department of Computer Science, P. G. Centre, Raichur for extending technical support.

\section{REFERENCES}

[1] Mohamed Amine KAFI, Yacinechallal, Djameldjenouri, Messaouddoudou, Abdelmadjid Bouabdallah, Nadjibbadache, "A Study of Wireless Sensor Networks for Urban Traffic Monitoring: Applications and Architectures", Elsevier, The $4^{\text {th }}$ Inter. Conf. on Ambient Systems, Networks and Technologies (ANT 2013), Procedia Computer Science, 19, 2013,pp. 617 - 626.

[2] M.Lavanya P. Muthukannan, Y.S.S. Bhargav, and V. Suresh, "IoT Based Automated Temperature and Humidity Monitoring and Control", Journal of Chemical and Pharmaceutical Sciences (JCHPS), special issue 5: October 2016, pp.86-88.

[3] Andrea Zanella, Lorenzo Vangelista, "Internet of Things for Smart Cities", IEEE Internet of Things Journal, vol 1,no. 1, Feb.2014, pp. 22-32.

[4] Mohd Ezwan Jalil, "Positioning and Location Tracking Using Wireless Sensor Network," Universiti Teknologi Malaysia, 2011

[5] Ding Libo and Wang Xuehmi "Design of a Web-based Temperature and Humidity Monitoring System", 2nd International Conference on Artificial Intelligence, Management Science and Electronic Commerce (AIMSEC), 2011, pp.2028-2030.

[6] Ali Akbar Siddiqui, Samreen Amir and Nimrah Ahmed, "Web-based Online Parameters monitoring and control system implemented on Raspberry Pi”, First Inter Conf. on Modern Communication \& Computing Technologies (MCCT'14), -28 February, 2014, Nawabshah, Pakistan.

[7] Akash, Amit and Birwal, "IoT-based Temperature and Humidity Monitoring System for Agriculture", Inter. Jour of Innovative Research in Science, Engineering and Technology, vol.6,issue 7, July 2017. Pp. 12756-12761.

[8] Bulipe Srinivas Rao, K. Srinivasa Rao, and N. Ome, "Internet of Things (IOT) Based Weather Monitoring system", Inter. Jour of Advanced Research in Computer and Communication Engineering, vol. 5, issue 9, September 2016, pp. 312-319.

[9] DHT11 Datasheet

[10] http://www.instructables.com/id/Arduino-Modules- RainSensor/

[11] http://www.resistorguide.com/photoresistor/

[12] P. Bhaskar and Malakondaiah, Experiments with Microcontrollers, I.K International Pvt. Ltd, 2010.

[13] Immanuel J., Parvathi C. S., P. Bhaskar, and L.S Sudheer, "Design and development of real time MATLAB-GUI based fuzzy logic controllers for DC motor speed control system", Int. Jour. of Electronics Engineering (IJEE), vol.3, no.1, pp.133-139, 2011.

[14] Immanuel J., Parvathi C. S., L. Shrimanth Sudheer, and P. Bhaskar, "Implementation of MATLAB-GUI based fuzzy logic controllers for liquid level control system", Int. Jour. of Electronics Engineering,(IJEE), vol.6, no.1, pp. 1-11, 2014. ISSN: 0973-7383. 


\section{AUTHOR DETAILS}

Immanuel J. received the M.Sc., M.Phil. and Ph.D. degrees in Instrumentation Technology from Gulbarga University, Gulbarga, KA, India, in 2005, 2008, and 2014 respectively.

Currently, he is a Guest Lecturer in the Department of Instrumentation Technology at Gulbarga University Post Graduate Centre, Raichur, KA, India. His research interests includes embedded systems, fuzzy logic control systems, MATLAB/DSP/PC based measurement, acquisition, and control. He has published and presented more than 16 papers in the journals of national/ international repute. He is a life member of the Instrument Society of India.

Rekha Patil received the M.Sc., degree in Computer Science from Gulbarga University, Gulbarga, KA, India, and M.Phil Computer Science from Vinayak Mission University, Salum, Tamil Nadu, India in 2005, and 2009 respectively.

Currently, she is a Guest Lecturer in the Department of Computer Science at Gulbarga University Post Graduate Centre, Raichur, KA, India. Her research interests include Discrete Mathematical structures, Data Structures, Software Engineering, java \& html programming. She has published and presented more than 04 papers in the journals of national/ international repute.

L. Shrimanth Sudheer received the M.Sc., M.Phil. and Ph.D degrees in Instrumentation Technology from Gulbarga University, Gulbarga, KA, India, in 2002, 2007, and 2014 respectively. Currently, he is a Guest Lecturer in the Department of Instrumentation Technology at Gulbarga University Post Graduate Centre, Raichur, KA, India. His research interests include embedded systems, fuzzy logic control systems, and PC based acquisition, measurement, and control. He has published and presented more than 30 papers in the journals of national/ international repute. He is a member of the Instrument Society of India, and associate of IETE, India.

Parvathi C. S. received the B.E. degree in Instrumentation Technology from PDA College of Engineering, Gulbarga University, KA, in 1981, M.Tech. degree in Instrumentation and Control Engineering from REC, Calicut, in 1983, and Ph.D. degree in Applied Electronics from Gulbarga University, Gulbarga, KA, India, in 2003. Currently, she is a Professor and Chairperson of the Department of Instrumentation Technology at Gulbarga University Post Graduate Centre, Raichur, KA, India. She has published more than 35 papers in journals of national/international repute. Her research interests include Fuzzy Control Systems, PC/DSP/ MATLAB based measurement, acquisition, and control, and process/ industrial control instrumentation. She is a member of ISTE and also the Instrument Society of India.

P. Bhaskar received the M.Sc., M.Phil., and Ph.D. degrees in Instrumentation from Sri Krishnadevaraya University, Anatapur, AP, India, in 1989, 1991, and 2001, respectively.

Currently, he is a Professor in the Department of Instrumentation Technology at Gulbarga University Post Graduate Centre, Raichur, KA, India. He has published more than 45 papers in journals of national/international repute. He has also authored a book on 8051 microcontroller. His research interests include Scientific/Analytical Instrumentation, Embedded Systems, and Intelligent Control Systems. He is a member of the Instrument Society of India. 